\title{
Shannon dimensionality of quantum channels and its application to photon entanglement
}

\author{
J.B. Pors 6 S.S.R. Oemrawsingh A. Aiello, M.P. van Exter, E.R. Eliel, G. W. 't Hooft, and J.P. Woerdman \\ Huygens Laboratory, Leiden University, P.O. Box 9504, 2300 RA Leiden, The Netherlands
}

\begin{abstract}
We introduce the concept of Shannon dimensionality $D$ as a new way to quantify bipartite entanglement as measured in an experiment. This is applied to orbital-angular-momentum entanglement of two photons, using two state analyzers composed of a rotatable angular-sector phase plate that is lens-coupled to a single-mode fiber. We can deduce the value of $D$ directly from the observed two-photon coincidence fringe. In our experiment, $D$ varies between 2 and 6 , depending on the experimental conditions. We predict how the Shannon dimensionality evolves when the number of angular sectors imprinted in the phase plate is increased and anticipate that $D \simeq 50$ is experimentally within reach.
\end{abstract}

PACS numbers: 03.67.Mn,42.50.Tx,42.65.Lm

Photons can be entangled in various degrees of freedom. The most extensively studied variety involves the polarization degrees of freedom, of which there are inherently two per photon. In a typical EPR-Bell type experiment, the state analyzers are polarizers, and when their relative orientation is scanned, this gives rise to a sinusoidal coincidence fringe [1]. This particular shape is characteristic of the two-dimensional nature of polarization entanglement.

Recently, much attention has been drawn to bipartite entanglement involving more than two degrees of freedom. With increasing dimensionality, quantum entanglement becomes correspondingly richer. Highdimensional entanglement is predicted to violate locality more strongly and to show more resilience to noise [2, 3]. From an applications perspective, it holds promise for implementing larger alphabets in quantum information, e.g. quantum cryptography [4], and for an increased security against eavesdropping [5]. High-dimensional entanglement can be studied employing the frequency-time [6] or position-momentum degrees of freedom, the latter having been demonstrated for both the transverse linear 7, 8 and orbital-angular-momentum degrees of freedom [9, 10].

It is crucial to have a quantifier of the dimensionality of entanglement as measured in an experiment [11]. In this Letter we introduce such a quantifier, using concepts from classical information theory in the spirit of Shannon [12]. We apply these ideas to orbital-angularmomentum entanglement, inserting appropriate angular state analyzers in the beamlines of a parametric downconversion setup. We have realized a Shannon dimensionality $2 \leq D \leq 6$ and we argue that $D \simeq 50$ is within reach.

In classical information theory [12], the number of independent communication channels of a signal is known

\footnotetext{
*Electronic address: pors@molphys.leidenuniv.nl

${ }^{\dagger}$ present address: Department of Physics, University of California, Santa Barbara, California 93106, USA
}

as the Shannon number. The signal being the state of a physical system, the Shannon number is also referred to as the number of degrees of freedom, or the number of modes, of that system [13, 14]. For example, a signal encoded in the polarization degrees of freedom of a light beam has a Shannon number equal to 2 .

When dealing with a bipartite quantum system in an entangled pure state $|\psi\rangle \in \mathscr{K}=\mathscr{K}_{A} \otimes \mathscr{K}_{B}$, the usual measure of the effective dimensionality of the Hilbert space in which the state lives, is given by the Schmidt number $K$ [15]

$$
K=\frac{1}{\operatorname{Tr}_{A}\left(\rho_{A}^{2}\right)}=\frac{1}{\operatorname{Tr}_{B}\left(\rho_{B}^{2}\right)} .
$$

Here, $\rho_{A}=\operatorname{Tr}_{B}(|\psi\rangle\langle\psi|)$ and $\rho_{B}=\operatorname{Tr}_{A}(|\psi\rangle\langle\psi|)$, are the reduced density matrices representing the states of the two sub-systems $A \in \mathscr{K}_{A}$ and $B \in \mathscr{K}_{B}$, respectively. Although a system may have infinitely many degrees of freedom, any actual measurement apparatus has effective access only to a finite number of them, say $D$. Such a dimensionality $D$ is referred to as the Shannon number of the measurement apparatus.

Consider an experiment measuring correlations between the two subsystems $A$ and $B$. There are two measuring apparatuses, say $\mathscr{P}_{A}(\alpha)$ and $\mathscr{P}_{B}(\beta)$, interacting with subsystems $A$ and $B$, respectively, where $\alpha$ and $\beta$ label possible settings of the two apparatuses. For a given setting $\xi \in\{\alpha, \beta\}$, detector $\mathscr{P}_{X}(\xi)$ is represented by the projection operator $\hat{\Gamma}(\xi)=|X(\xi)\rangle\langle X(\xi)|$, where $X \in\{A, B\}$, and $|X(\xi)\rangle$ is the state in which the system $X$ is left after measurement.

If a Von Neumann-type projective measurement is performed, the set of states $\{|X(\xi)\rangle\}_{\xi}$ obtained by varying $\xi$ is complete and orthonormal, namely

$$
\left\langle X(\xi) \mid X\left(\xi^{\prime}\right)\right\rangle=\delta_{\xi \xi^{\prime}}, \quad \sum_{\xi} \hat{\Gamma}(\xi)=\hat{1},
$$

where the measurement operators $\hat{\Gamma}(\xi)$ are Hermitean and idempotent. The number of these operators is equal to the dimension of the Hilbert space of the measured 
quantum system [16]. However, in many situations nonorthogonal measurements are made and Eqs. (2) do not hold [17]. In this case, the number of projection operators $\hat{\Gamma}(\xi)$ does not give the dimension of the Hilbert space of the measured system, and a new criterion must be introduced.

Let us therefore consider finite-dimensional systems, say $\operatorname{dim}\left(\mathscr{K}_{X}\right)=L$, and rewrite Eq. (2) for the case of non-orthogonal measurements as

$$
\left\langle X(\xi) \mid X\left(\xi^{\prime}\right)\right\rangle=g_{\xi \xi^{\prime}}, \quad \sum_{\xi} \hat{\Gamma}(\xi)=\hat{\gamma},
$$

where $G=\left[g_{\xi \xi^{\prime}}\right]$ is a matrix of size $L \times L$, and $\hat{\gamma}$ is an Hermitean operator. The eigenvalues $\gamma_{l}$ of $\hat{\gamma}$ give the detector's 'sensitivity' to the corresponding eigenmodes. In general, a detector will not be equally sensitive to all eigenmodes and some $\gamma_{l}$ are substantially larger than others. The effective dimensionality $D \leq L$ of the Hilbert space $\mathscr{D}$ where the measured system lives can be quantified as the Hilbert-Schmidt norm of the eigenvalue distribution [18]

$$
D \equiv \frac{1}{\operatorname{Tr}\left(\hat{\gamma}^{2}\right)}=\frac{1}{\sum_{l} \gamma_{l}^{2}}
$$

This dimensionality should be interpreted as the effective Shannon number of information channels [12, 13].

The isomorphism of Eq. (1) and Eq. (44) suggests a relation between the Schmidt number $K$ and the Shannon dimensionality $D$. The nature of such relation becomes clear if one notes that since the operators $\hat{\Gamma}(\xi)$ are Hermitian and positive semidefinite, the operator $\hat{\gamma}$ may be interpreted as a density matrix acting in $\mathscr{K}_{X}$ [19]. Thus, if we think of $\hat{\gamma}$ as a reduced density matrix of a bipartite system, then $K$ and $D$ are formally the same. However, it is important to note that while $K$ furnishes the dimensionality of the generated entanglement, $D$ gives the effective dimensionality of the space $\mathscr{D}$ that can potentially be probed and it is a property of the projection apparatus only. The dimensionality of the measured entanglement is a joint property of the generated system and analyzers, but simply amounts to $D$ as long as $\mathscr{K} \supset \mathscr{D}$.

Next, we apply our formal theory to an experiment on orbital-angular-momentum entanglement of two photons, in order to illustrate how detector characteristics bound the measured entanglement to an effective Shannon dimensionality $D$, while probing a generated state with Schmidt number $K \gg D$ (and $\mathscr{K} \supset \mathscr{D})$. Our experimental setup is depicted in Figure 1. Pumping a BBO non-linear crystal with a $150 \mathrm{~mW} \mathrm{Kr}^{+}$laser beam at $\lambda=413 \mathrm{~nm}$, we produce spatially entangled photons by means of spontaneous parametric down conversion. The state we generate is of the form $|\Psi\rangle=\sum_{l} \sqrt{\lambda_{l}}|l\rangle|-l\rangle$, where $|l\rangle$ denotes the orbital angular momentum eigenmode of order $l:\langle\phi \mid l\rangle=\exp (i l \phi) / \sqrt{2 \pi}$, with $\phi$ the azimuthal angle [20]. Employing Type-I collinear phase matching, we collect the full emission cone and with the experimental parameters of our setup (beam half-waist at the position of the crystal $w_{0}=250 \mu \mathrm{m}$ and crystal length $1 \mathrm{~mm}$ ) we obtain an azimuthal Schmidt number $K \simeq 31$ [21, 22, 23]. The twin photons are spatially separated by means of a non-polarizing beam splitter.

Each arm of the setup contains an angular state analyzer, composed of an angular phase plate that is lenscoupled to a single-mode fiber (see Fig. 11) 24]. The angular phase plates carry a purely azimuthal variation of the optical thickness. As in polarization entanglement [1], the phase plates are rotated around their normals and the photon coincidence rate is recorded as a function of their independent orientations [10].

The combined detection state of the two angularphase-plate analyzers, each acting locally, can be expressed as

$$
|A(\alpha)\rangle \otimes|B(\beta)\rangle=\left(\sum_{l} \sqrt{\gamma_{l}}|l\rangle e^{i l \alpha}\right)_{A} \otimes\left(\sum_{l} \sqrt{\gamma_{l}}|l\rangle e^{i l \beta}\right)_{B},
$$

where $\alpha$ and $\beta$ denote the orientations of the two phase plates, respectively [25]. The complex expansion coefficients $\sqrt{\gamma_{l}}$ are fixed by the physical profile of the angular phase plate and obey the normalization condition $\sum_{l}\left|\gamma_{l}\right|=1$. In general, the detection state constitutes a non-uniform superposition of orbital angular momentum modes. When the angular phase plates are rotated over $\alpha$ or $\beta$, all modes in the superposition rephase with respect to each other, yielding a set of detection states of the type Eq. (3). The effective Shannon dimensionality that is so probed is given by Eq. (4). It is the average number of modes captured by an analyzer when its phase plate is rotated over $360^{\circ}$.

As we have recently shown in Ref. [18], the Shannon dimensionality is straightforwardly deduced from the shape of the experimental coincidence curve; it is the inverse of the area underneath the peak-normalized coincidence

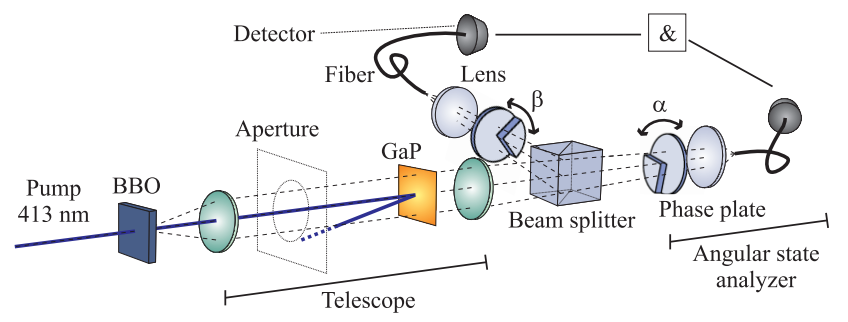

FIG. 1: (color online). Experimental setup. Orbital-angularmomentum entangled photons are emitted at $826 \mathrm{~nm}$ by a BBO crystal, cut for Type-I collinear phase matching. A thin GaP wafer serves to eliminate the pump beam. The two-photon field can be clipped with an aperture. The twinphotons are spatially separated by a beam splitter and imaged on the angular phase plates $\left(f_{2}=4 f_{1}=40 \mathrm{~cm}\right)$. Just behind the phase plates, the frequency-degenerate photons are selected by interference filters (not shown), centered around $826 \mathrm{~nm}$ with a $10 \mathrm{~nm}$ width. The phase plates (shown are quarter-sector plates) are oriented at angles $\alpha$ and $\beta$, and photon counts are rendered by a coincidence circuit. 
fringe, obtained when rotating one of the phase plates.

In our experiment, we have used angular-sector phase plates; these have a single arc sector, characterized by the angle $\delta$, whose optical thickness is $\lambda / 2$ greater than that of the remainder of the plate [24]. The part of the field that crosses this sector thus flips sign. The phase plates are manufactured from fused-quartz plane-parallel plates, having a wedge angle of $0.25 "$ ". They are processed by a combination of photolithography, wet etching, deposition and lift-off, resulting in a well-defined mesa structure, with a transition region that is typically $20 \mu \mathrm{m}$ wide. The insets of Figure 2 show two such plates; a half-sector plate $(\delta=\pi)$ consisting of two equal halves that are phase shifted by $\pi$; and a quarter-sector plate $(\delta=\pi / 2)$ having one quadrant $\pi$-phase shifted with respect to the remainder of the plate.

For state analyzers that are equipped with such sector phase plates, the Shannon dimensionality is given by [18]

$$
D(\delta)= \begin{cases}{\left[1-4 \frac{\delta}{\pi}+6\left(\frac{\delta}{\pi}\right)^{2}-\frac{8}{3}\left(\frac{\delta}{\pi}\right)^{3}\right]^{-1},} & \delta \in[0, \pi], \\ D(2 \pi-\delta), & \delta \in[\pi, 2 \pi] .\end{cases}
$$

For $\delta=0$ we find the trivial result $D=1$; a planar plate does nothing. For $\delta=\pi$, i.e. a state analyzer equipped with a half-sector plate, we arrive at $D=3$. For an analyzer equipped with a quarter-sector plate we find $D=6$. This is the maximum value for a single angular-sector phase plate. We note that for our setup indeed $K \gg D$.

In the experiment, we scan one angular-sector phase plate over a $360^{\circ}$ rotation, the other remaining fixed, and measure the coincidence rate. In terms of Klyshko's picture of advanced waves [26], valid when $K \gg D$, the resulting shape of the coincidence curves can be explained in terms of the mode overlap of the two state analyzers. Figure2(a) shows experimental results obtained with two half-sector plates $(\delta=\pi)$, having step height of $0.48 \lambda$. The data points form a double parabolic fringe, consistent with theory (solid curve). The maxima at $0^{\circ}$ and $180^{\circ}$ are sharply peaked. The zeros of the fringe are very deep; less than 10 counts per 10 seconds. The maximum coincidence rate is of the order of $6.5 \times 10^{3}$ per $10 \mathrm{sec}-$ onds, compared to $10^{5}$ single counts. We verified that the coincidence rate depends on the relative orientation between the two phase plates only, the fringe visibility being $>99 \%$ for all cases studied. This basis independence is the key aspect of quantum entanglement. From the area underneath the data we deduce the experimental value $D=3.0$. Note that a parabolic fringe was also reported in Ref. [10], obtained with non-integer spiral phase plates. We conclude that also in that case $D=3$.

An aperture, positioned inside the telescope, allows us to control the number of detected modes (see Figure 1). Because of the anti-symmetric profile of the half-sector plate, the detection state contains only odd expansion terms (see Eq. (5)) in a fashion $\gamma_{l}=\gamma_{-l}$. When the aperture size is reduced, higher-order orbital-angularmomentum modes are cut off so that, eventually, only the modes $l=1$ and $l=-1$ survive. We then expect a sinusoidal fringe, analogous to two-dimensional polarization entanglement [1]. In the experiment, we observe that the coincidence curve is gradually transformed from parabolic to sinusoidal when the aperture gets smaller. Using an aperture of $600 \mu \mathrm{m}$ diameter, we are in an intermediate regime (squares, $D=2.1$ ), while using a 400 $\mu \mathrm{m}$ diaphragm yields a curve that resembles a sine very well (triangles, $D=2.0$ ). The dashed and dotted curve are theoretical predictions.

To achieve $D=6$, we use two quarter-sector plates $(\delta=\pi / 2)$, carrying an edge discontinuity deviating less than $3 \%$ from $\lambda / 2$. The circles in Figure 2(b) show our experimental results, revealing a coincidence curve which is parabolic for $|\alpha-\beta| \leq 90^{\circ}$ and equal to zero otherwise, in agreement with theory (solid curve). We find $D=$ 5.8 , in very good agreement with the expected value of 6 mentioned above.

The maximum value of the Shannon dimensionality that can be achieved with a phase plate having but a single sector is $D=6$. Can one reach higher values of $D$

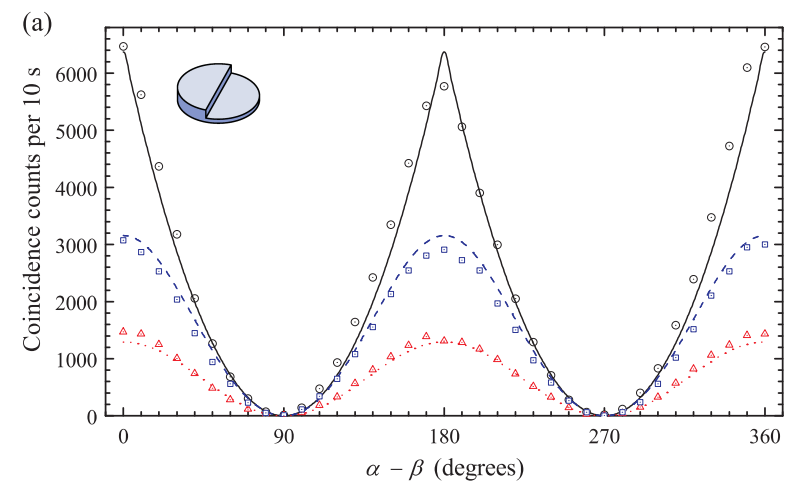

(b)

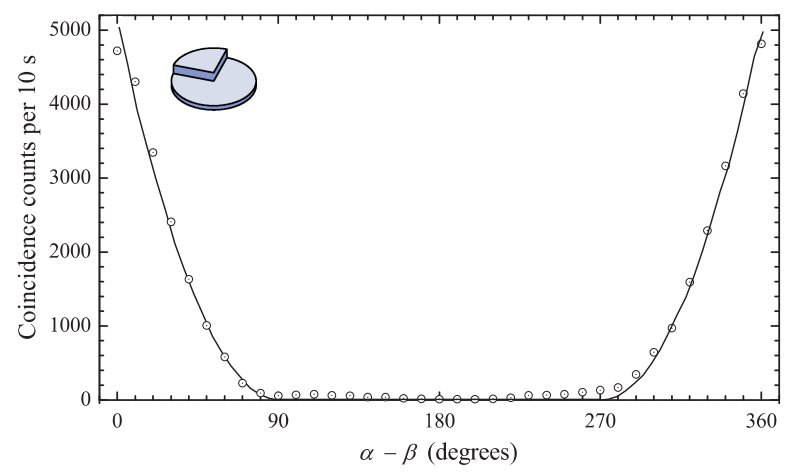

FIG. 2: (color online). Coincidence count rate vs. the relative orientation of the two state analyzers. Points denote experimental data, the curves are theoretical predictions. (a) Halfsector plate. The parabolic fringe (circles) is a signature of a dimensionality larger than two: we find $D=3.0$. Truncating the number of modes, by closing the aperture, gradually reduces the parabola into a sine of dimensionality 2.0 (triangles). (b) Quarter-sector plate. The piece-wise parabolic fringe yields an experimental dimensionality of 5.8 (circles), where theory predicts $D=6$. 
by using plates with more sectors? To answer this question, we consider plates with $N$ sectors that are phase shifted by $\pi$ with respect to interjacent regions. For each choice of sector angles, we calculate the expansion coefficients $\left\{\sqrt{\gamma_{l}}\right\}$ and, subsequently, $D$ (see Eq. (4) and (5)). Next, we maximize $D$ by adjusting the sector angles using a Monte-Carlo random-search algorithm. The result is plotted in Figure 3. showing a graph of the maximum value of $D$ versus the number of mesas $N$. For $10 \mathrm{such}$ sectors, we find $D=49.9$. The insets show the optimal phase plates for $N=1$ (quarter-sector plate), $N=2$, and $N=3$.

In conclusion, we have introduced the effective Shannon dimensionality as a novel quantifier of entanglement as measured in an actual experiment. We have demonstrated its significance to the case of two-photon orbitalangular-momentum entanglement. Using angular-sector phase plates, we have achieved Shannon dimensionalities up to $D=6$. We anticipate that it is feasible to probe dimensionalities as high as 50, using multi-sector phase plates. These can be manufactured by means of photoor e-beam lithography as in diffractive-optics technology. Alternatively, the use of adaptive optical devices, such as spatial light modulators or micro-mirror arrays, seems promising, particularly because of their versatility with regard to plate patterns. However, the ultimate limit to the Shannon dimensionality is constrained by the angular Schmidt number of the source; using periodically poled crystals, such as PPKTP, $K \sim 100$ is viable for realistic values of pump-beam waist and crystal length, without loss of count rates [27].

This work was supported by the Stichting voor Fundamenteel Onderzoek der Materie.

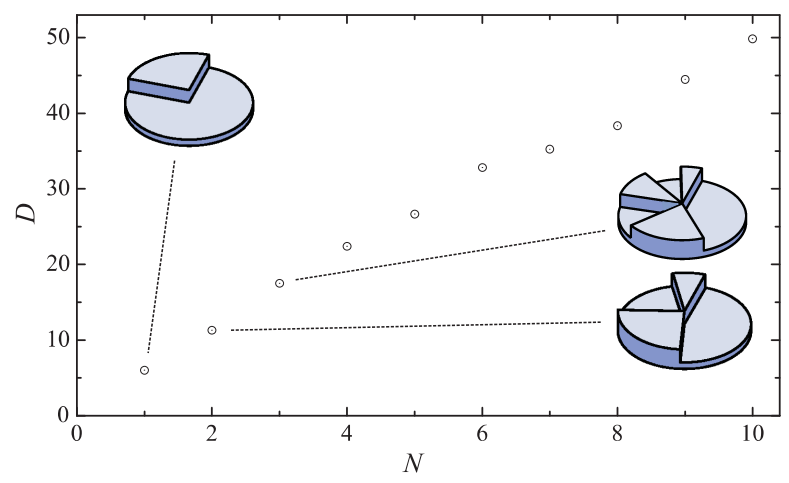

FIG. 3: (color online). Maximum dimensionality that can be accessed with sector phase plates having $2 N$ angular sectors alternatingly phase shifted by $\pi$. The insets show the optimized plates for $N=1, N=2$ and $N=3$.
[1] A. Aspect, P. Grangier, and G. Roger, Phys. Rev. Lett. 47, 460 (1981).

[2] D. Collins, N. Gisin, N. Linden, S. Massar, and S. Popescu, Phys. Rev. Lett. 88, 040404 (2002).

[3] D. Kaszlikowski, P. Gnaciński, M. Żukowski, W. Miklaszewski, and A. Zeilinger, Phys. Rev. Lett. 85, 4418 (2000).

[4] H. Bechmann-Pasquinucci and W. Tittel, Phys. Rev. A 61, 062308 (2000).

[5] L. Zhang, C. Silberhorn, and I. A. Walmsley, Phys. Rev. Lett. 100, 110504 (2008).

[6] H. de Riedmatten, I. Marcikic, H. Zbinden, and N. Gisin, Quant. Inf. Comp. 2, 425 (2002).

[7] L. Neves, G. Lima, J. G. Aguirre Gómez, C. H. Monken, C. Saavedra, and S. Pádua, Phys. Rev. Lett. 94, 100501 (2005).

[8] M. N. O'Sullivan-Hale, I. A. Khan, R. W. Boyd, and J. C. Howell, Phys. Rev. Lett. 94, 220501 (2005).

[9] A. Mair, G. W. A. Vaziri, and A. Zeilinger, Nature 412, 313 (2001).

[10] S. S. R. Oemrawsingh, X. Ma, D. Voigt, A. Aiello, E. R. Eliel, G. W. 't Hooft, and J. P. Woerdman, Phys. Rev. Lett. 95, 240501 (2005).

[11] N. Brunner, S. Pironio, A. Acín, N. Gisin, A. A. Méthot, and V. Scarani, Phys. Rev. Lett. 100, 210503 (2008).

[12] C. E. Shannon and W. Weaver, The Mathematical Theory of Communication (University of Illinois Press, New York, 1949).

[13] G. T. di Francia, J. Opt. Soc. Am. 59, 799 (1969).

[14] F. Gori and G. Guattari, Opt. Comm. 7, 163 (1973).

[15] M. U. Karelin, Opt. Spectrosc. 103, 193 (2007).
[16] H. E. Brandt, Am. J. Phys 67, 434 (1999).

[17] R. T. Thew, K. Nemoto, A. G. White, and W. J. Munro, Phys. Rev. A 66, 012303 (2002).

[18] J. B. Pors, A. Aiello, S. S. R. Oemrawsingh, M. P. van Exter, E. R. Eliel, and J. P. Woerdman, Phys. Rev. A 77, 033845 (2008).

[19] I. Bengtsson and K. Życzkowski, Geometry of quantum states: An introduction to quantum entanglement (Cambridge University Press, 2006).

[20] S. P. Walborn, A. N. de Oliveira, R. S. Thebaldi, and C. H. Monken, Phys. Rev. A 69, 023811 (2004).

[21] C. K. Law and J. H. Eberly, Phys. Rev. Lett. 92, 127903 (2004).

[22] M. P. van Exter, A. Aiello, S. S. R. Oemrawsingh, G. Nienhuis, and J. P. Woerdman, Phys. Rev. A 74, 012309 (2006).

[23] M. P. van Exter, P. S. K. Lee, S. Doesburg, and J. P. Woerdman, Opt. Express 15, 6431 (2007).

[24] S. S. R. Oemrawsingh, J. A. de Jong, X. Ma, A. Aiello, E. R. Eliel, G. W. 't Hooft, and J. P. Woerdman, Phys. Rev. A 73, 032339 (2006).

[25] The set of OAM eigenmodes constitutes the appropriate basis to expand the detection state, for these modes are eigenfunctions of rotation over $\alpha$, and the set contains the fiber mode $l=0$.

[26] A. V. Belinskii and D. N. Klyshko, Zh. Eksp. Teor. Fiz. 105, 487 (1994).

[27] M. Fiorentino, S. M. Spillane, R. G. Beausoleil, T. D. Roberts, P. Battle, and M. W. Munro, Opt. Expr. 15, 7479 (2007). 